\title{
Collective excitations of composite fermions across multiple $\Lambda$ levels
}

\author{
Dwipesh Majumder', Sudhansu S. Mandal' and Jainendra K. Jain ${ }^{2 \star}$
}

The fractional quantum Hall state ${ }^{1}$ is a quintessential system for the study of collective quantum behaviour. In such a system, the collective behaviour results in the creation of so-called composite fermions, quasi-particles formed by electrons attached to magnetic flux quanta. Recently, a new collective mode was unexpectedly observed in Raman scattering experiments ${ }^{2}$ on such a system as it was found to split off from the familiar 'fundamental' long-wavelength mode on increase of the wave vector. Here, we present results from extensive theoretical calculations that make a compelling case that this mode corresponds to an excitation of a composite fermion across two $\Lambda$ levels-effective kinetic energy levels resembling Landau levels for such particles. In addition to explaining why this excitation merges with the fundamental mode in the long-wavelength limit, our theory also provides a good quantitative account of the amount of splitting, and makes several experimentally verifiable predictions.

Unlike the well-known quantum phenomena of superfluidity and superconductivity, the fractional quantum Hall effect ${ }^{1}$ does not entail any Bose-Einstein condensation but occurs as a result of the formation of topological electron-vortex bound states called composite fermions ${ }^{3}$. Transport ${ }^{4}$, light scattering ${ }^{5,6}$ and phonon scattering ${ }^{7,8}$ have been extensively used during the past quarter of a century to probe its numerous excitations. Of particular significance is the neutral collective mode, which was first studied theoretically at Landau-level filling $v=1 / 3$ in a single-mode approximation ${ }^{9}$, wherein, following Feynman's theory of the phonon-roton mode of helium superfluid, the excitation is modelled as a density wave. The neutral collective mode was detected by Raman scattering ${ }^{5}$, with the observations generally consistent with the predictions of the single-mode approximation in the long-wavelength limit. More recently, however, Hirjibehedin et al. ${ }^{2}$ have discovered that this mode is not a single mode, as believed earlier, but splits into two as the wave vector is increased. By definition, the single-mode approximation cannot accommodate a doublet. A hydrodynamic approach has been proposed ${ }^{10}$ to account for the experimental observation, but does not take into account the microscopic physics of the fractional quantum Hall effect (FQHE), does not naturally explain the merging of the two modes in the long-wavelength limit and also greatly underestimates the splitting.

We show here that this new mode finds a natural explanation within the composite-fermion theory ${ }^{3}$. Composite fermions are bound states of electrons and an even number $(2 p)$ of quantized vortices. Because of the Berry phases produced by the bound vortices, composite fermions effectively experience a much reduced magnetic field $B^{*}=B-2 p \rho \phi_{0}$ ( $B$ is the external magnetic field, $\rho$ is the two-dimensional density and $\phi_{0}=h c / e$ is called the flux quantum). Composite fermions form their own Landau-like kinetic energy levels in this reduced magnetic field, called $\Lambda$ levels, and their filling factor $v^{*}$ is related to the electron filling factor $v$ through the relation $v=v^{*} /\left(2 p v^{*}+1\right)$. In particular, at $v=n /(2 p n+1)$, the ground state consists of $n$ filled $\Lambda$ levels. In the composite-fermion theory, the lowest-energy neutral excitation is a particle-hole pair, or an exciton, of composite fermions, wherein a single composite fermion from the topmost occupied $\Lambda$ level is excited into the lowest unoccupied $\Lambda$ level (Fig. $1 \mathrm{~b}$ shows the fundamental composite-fermion exciton at $v=2 / 5$ ). The validity of this description has been confirmed for fractions of the form $v=n /(2 p n+1)$ by comparison to exact diagonalization results as well as to experiment ${ }^{11,12}$. This physical explanation for the neutral collective excitations is distinct from the single-mode approximation, and, in particular, suggests the possibility of extra collective modes, in which a composite fermion is excited across two or more $\Lambda$ levels, as shown schematically in Fig. 1c, in complete analogy to the collective modes of an integral quantum Hall state $^{13}$. However, it is far from obvious that the composite-fermion collective modes across different $\Lambda$ levels should merge in the longwavelength limit. In fact, a model that takes composite fermions as non-interacting produces collective modes spaced by the effective cyclotron energy in the long-wavelength limit, as also found for the dispersions obtained in the composite-fermion Chern-Simons approach ${ }^{14,15}$; if correct, this would make such physics irrelevant to the new collective mode discovered in ref. 2. For a more definitive test, however, the composite-fermion exciton-mode spectrum must be evaluated in a microscopic approach that includes effects of inter-composite-fermion interactions.

Exact diagonalization studies of the FQHE state do not by themselves provide an understanding of the underlying physics, and are not useful in the present context, because, as seen below, systems as large as 200 particles are required for investigating the experimentally relevant wave vectors; the Fock space increases exponentially with the number of particles, and at present exact diagonalization is possible only for $10-12$ particles for the filling factors of interest here. Our quantitative investigations below exploit accurate trial wavefunctions for composite fermions ${ }^{3}$. The standard spherical geometry is used in our calculations, which considers electrons moving on the surface of a sphere, subjected to a radial magnetic field. The magnetic field can be thought to emanate from a 'magnetic monopole' of strength $Q$ at the centre, which produces a total magnetic flux of $2 Q \phi_{0}$ through the surface of the sphere. This maps into a system of composite fermions at an effective flux $Q^{*}=Q-N+1$, with $Q$ chosen so that the state at $Q^{*}$ is an integral quantum Hall state at filling $v^{*}=n$. The wavefunction $\Psi^{\mathrm{CF}-\mathrm{g}}$ for the FQHE ground state at $v=n /(2 n+1)$ is obtained by composite-fermionizing the $v^{*}=n$ integral quantum Hall state $\Phi^{\mathrm{g}}$. To model neutral collective excitations, we first construct wavefunctions of the excitons of the

${ }^{1}$ Department of Theoretical Physics, Indian Association for the Cultivation of Science, Jadavpur, Kolkata 700 032, India, ${ }^{2} 104$ Davey Laboratory, Physics Department, Pennsylvania State University, University Park, Pennsylvania 16802, USA. *e-mail: jain@phys.psu.edu. 


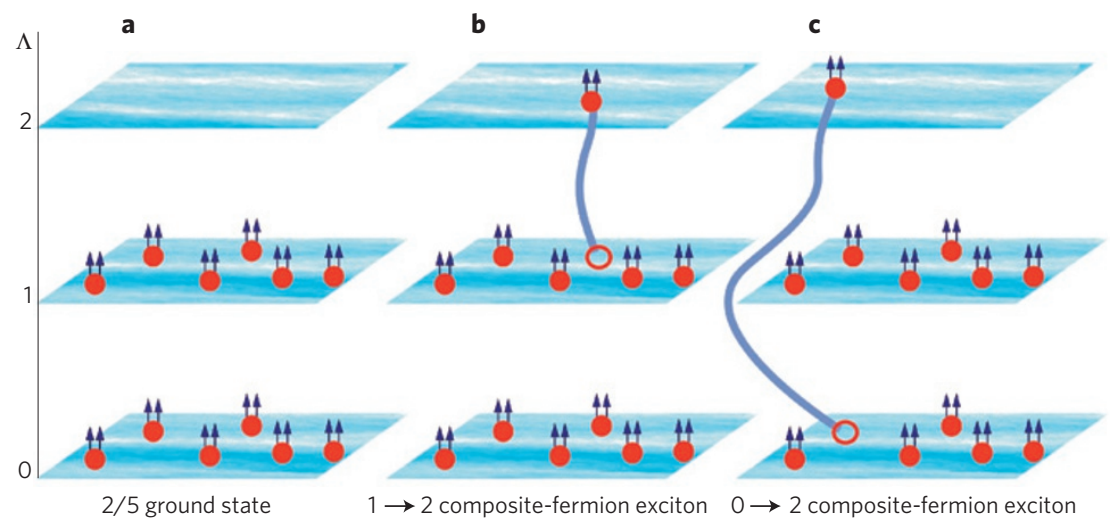

Figure 1 | Schematic diagram of composite-fermion excitons. Each composite fermion is shown as an electron carrying vortices represented by arrows. a, Representation of the ground state at $v=2 / 5$ as two filled $\Lambda$ levels. b,c, $1 \rightarrow 2$ (b) and $0 \rightarrow 2$ (c) composite-fermion excitons.
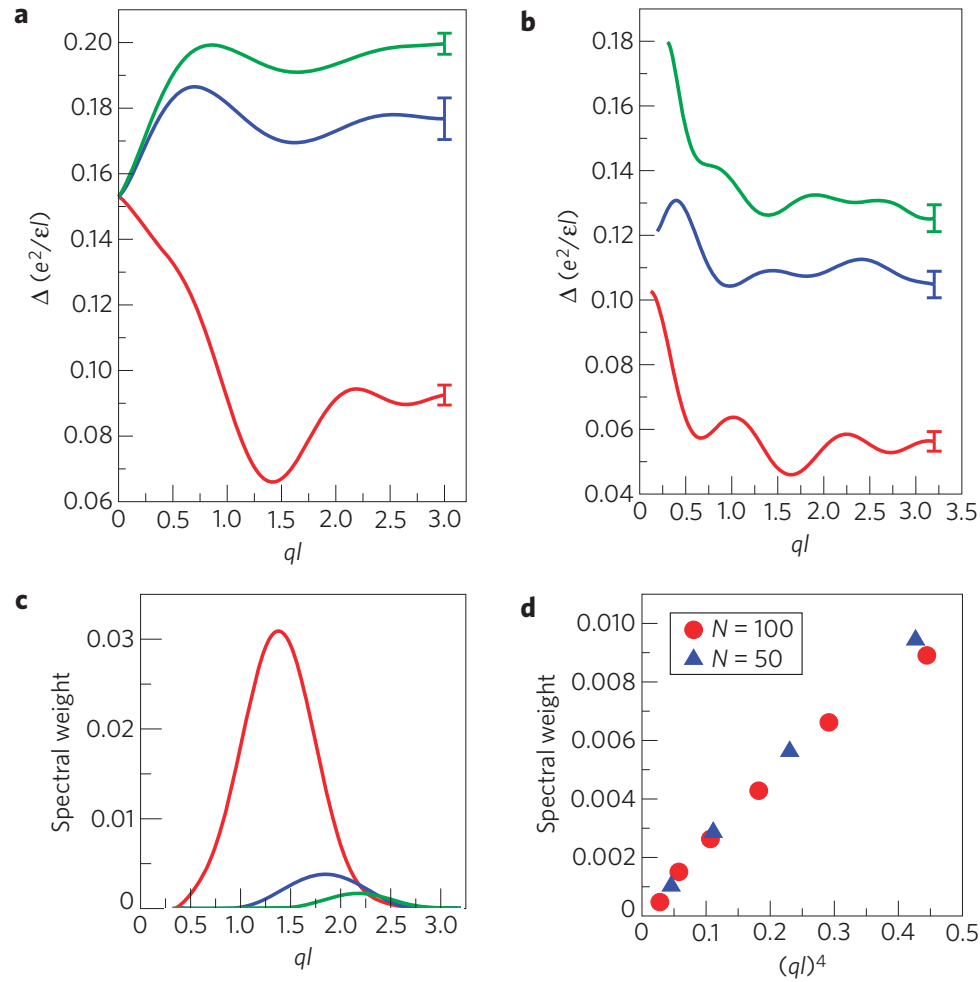

Figure 2 | Dispersions of several composite-fermion excitons and their spectral weights. a, The three lowest composite-fermion exciton modes at $v=1 / 3$, obtained from $0 \rightarrow 1,0 \rightarrow 2$ and $0 \rightarrow 3$ excitons. The error bar at the end of each curve represents the typical statistical error in the energy determined by the Monte Carlo method. The energies are quoted in units of $e^{2} / \epsilon \ell$, where $\epsilon$ is the dielectric constant of the background semiconductor and $\ell=\sqrt{\hbar c / e B}$ is the magnetic length. $\mathbf{b}$, The three lowest composite-fermion exciton modes at $v=2 / 5$, obtained from $1 \rightarrow 2,0 \rightarrow 2$ and $1 \rightarrow 3$ excitons. $\mathbf{c}$, Spectral weights for the three modes at $v=1 / 3$ for $N=100$. The curves from top to bottom correspond to the three modes shown in a respectively from bottom to top. $\mathbf{d}$, The spectral weight for the lowest-energy composite-fermion exciton at $v=1 / 3$ at small $q \ell$ for $N=50$ and $N=100$. All results in this figure are for the Coulomb eigenstates $\chi_{\lambda}^{\text {CF-ex. }}$

integral quantum Hall state, denoted by $\left\{\Phi_{\lambda, L}^{\mathrm{ex}}\right\}$, where $L$ is the total orbital angular momentum of the exciton and $\lambda$ labels different excitons of the type shown in Fig. 1. We composite-fermionize this basis to obtain $\left\{\Psi_{\lambda, L}^{\mathrm{CF}-\mathrm{ex}}\right\}$, which gives a set of basis functions for composite-fermion excitons. We orthonormalize this basis and diagonalize the Coulomb Hamiltonian to obtain the energies of the physical excitations, and also their wavefunctions $\left\{\chi_{\lambda, L}^{\mathrm{CF}-\mathrm{ex}}\right\}$. The scalar products of various basis functions and the Hamiltonian matrix elements are evaluated by the Metropolis Monte Carlo method. Blocks of different $L$ are not coupled by the interaction, so can be diagonalized separately. More details can be found in refs 16, 17 and Supplementary Information.
Even though our immediate interest is in understanding the splitting of the collective mode at $v=1 / 3$, we consider, for completeness, the three lowest collective modes at two filling factors: $0 \rightarrow 1,0 \rightarrow 2$ and $0 \rightarrow 3$ modes at $v=1 / 3$, and $1 \rightarrow 2$, $1 \rightarrow 3$ and $0 \rightarrow 2$ modes at $v=2 / 5$. We have studied systems with 50, 100 and 200 particles at both $v=1 / 3$ and $v=2 / 5$. The results shown in Fig. 2a, b refer to the 200-particle system, which we believe accurately represents the thermodynamic limit. The exciton dispersions are quoted as a function of the wave vector $q$, defined as $q=L / R$, where $R=\sqrt{Q}$ is the radius of the sphere in the unit of magnetic length $\ell=\sqrt{\hbar c / e B}$. The dispersion curves are obtained by averaging over $1.2 \times 10^{7}$ Monte Carlo iterations. 


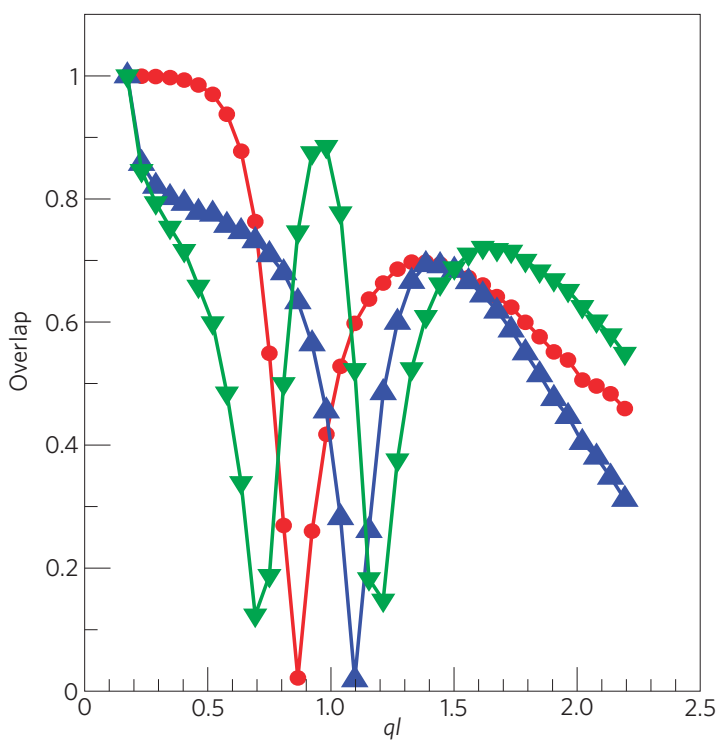

Figure 3 | Overlaps between the 'unorthogonalized' exciton basis states. Overlaps $\left\langle\Psi_{1, L}^{\mathrm{CF}-e x} \mid \Psi_{2, L}^{\mathrm{CF}-e x}\right\rangle$ (red circles), $\left\langle\Psi_{1, L}^{\mathrm{CF}-\mathrm{ex}} \mid \Psi_{3, L}^{\mathrm{CF}-e x}\right\rangle$ (blue uptriangles) and $\left\langle\Psi_{2, L}^{\mathrm{CF}-\mathrm{ex}} \mid \Psi_{3, L}^{\mathrm{CF} \text {-ex }}\right\rangle$ (green downtriangles), where $\Psi_{\lambda, L}^{\mathrm{CF} \text {-ex }}$ are compositefermion exciton wavefunctions at $v=1 / 3$ before orthogonalization. (The dispersions in Fig. 2a are obtained after orthogonalization of these modes and diagonalizing the Coulomb Hamiltonian in the orthogonal basis.) The overlaps are evaluated for $N=200$.

The qualitative and quantitative features of experiment at $v=1 / 3$ are nicely reproduced by our theory. Most strikingly, in Fig. 2a all modes are seen to merge in the long-wavelength limit. We note that this does not require any fine tuning of parameters (the wavefunctions $\Psi^{\mathrm{CF}-\mathrm{g}}$ and $\Psi^{\mathrm{CF}-\mathrm{ex}}$ do not contain any adjustable parameters) but is a robust effect in the compositefermion theory. To gain an insight into this merging, we compute the overlap between $\Psi_{1, L}^{\mathrm{CF}-e x}, \quad \Psi_{2, L}^{\mathrm{CF}-\mathrm{ex}}$ and $\Psi_{3, L}^{\mathrm{CF}-\mathrm{ex}}$ at $v=1 / 3$, shown in Fig. 3 for $N=200$; surprisingly, the overlaps approach unity at small $L$, becoming precisely equal to 1 for $L=2$ and $L=3$. (A comparison is not possible for $L=1$ because, in the spherical geometry, the smallest total orbital angular momentum available for the $n \rightarrow n+\lambda$ exciton is $\lambda$.) We have tested this result for many system sizes and believe that it holds in the thermodynamic limit. This demonstrates that all three modes become identical at small wave vectors, explaining why they merge into a single mode at small wave vectors; the single mode at $q \ell=0$ is to be distinguished from two degenerate modes. When the wavefunctions are linearly independent, multiple modes are produced after orthogonalization.

Even though the composite-fermion exciton states obtained from orthogonal collective modes of the integral quantum Hall effect become exactly equal at $L=2$ and 3 in our numerical study, we have not succeeded in deriving this result analytically. The wavefunctions are very complicated and the lowest-Landau-level projection does not lend itself to simple analytical treatments. We note that a Fock space reduction for excited states in going from the integral quantum Hall effect to the fractional quantum Hall effect has been found previously in another related context: for total orbital angular momentum $L=1$, the wavefunction for the $0 \rightarrow 1$ composite-fermion excitation is identically annihilated by the lowest-Landau-level projection operator ${ }^{11}$.

For a quantitative confirmation, we compare the theoretical splitting to the observed one. The light scattering experiments probe only very small wave-vector exchanges, but with our large systems we are able to make meaningful quantitative comparisons. The experimental splitting at $q \ell=0.15$, which is the largest wave vector accessible in experiments, is approximately 0.009(3) $e^{2} /(\epsilon \ell)$. With 200 particles, the smallest wave vector directly accessible is $q \ell=0.17$, but from a smooth extrapolation of the theoretical dispersion (assuming convergence at $q \ell \rightarrow 0$ ), we obtain at $q \ell=0.15$ a splitting of $0.013(5) e^{2} /(\epsilon \ell)$, which is in very good agreement with experiment, especially given the smallness of the energy difference and the neglect, in our calculations, of disorder, Landau-level mixing and finite-width effects, which are all expected to slightly reduce the splitting.

The Raman intensity is proportional to the spectral weight, given by $S_{q}=(1 / N)\left|\left\langle\chi_{\lambda, L}^{\text {ex }}\left|\rho_{L}\right| \Psi^{\mathrm{CF}-\mathrm{g}}\right\rangle\right|^{2}$, where the density operator at $q=L / R$ is defined as $\rho_{L}=\sum_{i} Y_{L 0}\left(\theta_{i}, \phi_{i}\right)$ in terms of spherical harmonics. Figure $2 \mathrm{c}$ shows our calculated spectral weights for various orthogonal modes, suggesting that the collective modes involving excitations across several $\Lambda$ levels are expected to be weaker than the fundamental mode. It is well known ${ }^{9}$ that in the long-wavelength limit the leading term in the spectral weight, proportional to $(q \ell)^{2}$, is exhausted by the inter-Landau-level Kohn mode at the cyclotron energy. We have explicitly verified that the spectral weight of the lowest mode at $v=1 / 3$ goes as $(q \ell)^{4}$ at small wave vectors (Fig. $2 \mathrm{~d}$ ), which serves as an independent test of the accuracy of the method. We believe that the same is true for the other modes at $v=1 / 3$ as well, but have not confirmed it explicitly because their much smaller spectral weights would require substantially more computational time. In spite of the small spectral weight, the intra-Landau-level collective modes can be observable in resonant Raman scattering owing to a strong resonant enhancement of the matrix elements ${ }^{18}$, although a detailed theory is not yet available.

The relation of the results presented above to the so-called two-roton mode is worth discussing. Motivated by a discrepancy between the experimental and theoretical energies in the longwavelength limit ${ }^{5,12}$, previous theoretical studies modelled the longwavelength mode as a two-roton bound state ${ }^{9,19,20}$, which was shown in explicit calculations ${ }^{19,20}$ to have a slightly lower energy than the single exciton, bringing the theoretical energy closer to the experimental one. The two-roton mode can also be interpreted as the single exciton mode hybridized with excitations consisting of two composite-fermion excitons; this is a natural interpretation given that the energy of the two-roton bound state is close to the single exciton energy (in the long-wavelength limit) and the wavefunctions of the two are not orthogonal. The physics discussed in the present work is distinct, in the sense that whereas the two-roton physics deals with corrections to the energy of the fundamental mode in the long-wavelength limit, the present work is concerned with the splitting of that mode at finite wave vectors. In fact, a mixing with excitations consisting of two composite-fermion excitons will lower the energies of all of the collective modes considered here, but we have not studied that effect because it is unlikely to alter the amount of the splitting significantly.

The results presented above have a number of experimental implications. The collective mode at $v=1 / 3$ is in fact seen to split into several modes (rather than just two), although the higher modes are very close in energy at small wave vectors and may not be readily resolvable. Multiple modes are predicted also at $v=2 / 5$. However, in contrast to $v=1 / 3$, the two lowest modes at $v=2 / 5$ do not merge in the long-wavelength limit; the composite-fermion theory thus predicts an absence of splitting of the long-wavelength collective mode at $v=2 / 5$. (This is to be contrasted with the hydrodynamic approach ${ }^{10}$, which obtains similar behaviour for all fractions.) Our theory also obtains the full dispersion of the new collective modes, which is outside the range of Raman experiments, but possibly observable in photoluminescence experiments in the presence of a 'grating' produced by piezoelectric coupling to certain frozen-in phonons, which picks out certain wave vectors in absorption spectra ${ }^{21}$. 
Received 13 November 2008; accepted 14 April 2009; published online 10 May 2009

\section{References}

1. Tsui, D. C., Stormer, H. L. \& Gossard, A. C. Two-dimensional magnetotransport in the extreme quantum limit. Phys. Rev. Lett. 48 1559-1562 (1982).

2. Hirjibehedin, C. F. et al. Splitting of long-wavelength modes of the fractional quantum Hall liquid at $v=1 / 3$. Phys. Rev. Lett. 95, 066803 (2005).

3. Jain, J. K. Composite fermion approach for the fractional quantum Hall effect. Phys. Rev. Lett. 63, 199-202 (1989).

4. Du, R. R., Stormer, H. L., Tsui, D. C., Pfeiffer, L. N. \& West, K. W. Experimenta evidence for new particles in the fractional quantum Hall effect. Phys. Rev. Lett. 70, 2944-2947 (1993).

5. Pinczuk, A., Dennis, B. S., Pfeiffer, L. N. \& West, K. W. Observation of collective excitations in the fractional quantum Hall effect. Phys. Rev. Lett. 70, 3983-3986 (1993)

6. Kang, M., Pinczuk, A., Dennis, B. S., Pfeiffer, L. N. \& West, K. W. Observation of multiple magnetorotons in the fractional quantum Hall effect. Phys. Rev. Lett. 86, 2637-2640 (2001).

7. Mellor, C. J. et al. Phonon absorption at the magnetoroton minimum in the fractional quantum Hall effect. Phys. Rev. Lett. 74, 2339-2342 (1995).

8. Zeitler, U. et al. Ballistic heating of a two-dimensional electron system by phonon excitation of the magnetoroton minimum at $v=1 / 3$. Phys. Rev. Lett. 82, 5333-5336 (1999).

9. Girvin, S. M., MacDonald, A. H. \& Platzman, P. M. Collective-excitation gap in the fractional quantum Hall effect. Phys. Rev. Lett. 54, 581-583 (1985).

10. Tokatly, I. V. \& Vignale, G. New collective mode in the fractional quantum Hall liquid. Phys. Rev. Lett. 98, 026805 (2007).

11. Dev, G. \& Jain, J. K. Band structure of the fractional quantum Hall effect. Phys. Rev. Lett. 69, 2843-2846 (1992).

12. Scarola, V. W., Park, K. \& Jain, J. K. Rotons of composite fermions: Comparison between theory and experiment. Phys. Rev. B 61, 13064-13072 (2000).
13. Kallin, C. \& Halperin, B. I. Excitations from a filled Landau-level in the two-dimensional electron-gas. Phys. Rev. B 30, 5655-5668 (1984).

14. Lopez, A. \& Fradkin, E. Response functions and spectrum of collective excitations of fractional-quantum-Hall-effect systems. Phys. Rev. B 47, 7080 (1993).

15. Simon, S. H. \& Halperin, B. I. Finite-wave-vector electromagnetic response of fractional quantized Hall states. Phys. Rev. B 48, 17368 (1993).

16. Jain, J. K. \& Kamilla, R. K. Composite fermions in the Hilbert space of the lowest electronic Landau level. Int. J. Mod. Phys. B 11, 2621-2660 (1997).

17. Mandal, S. S. \& Jain, J. K. Theoretical search for the nested quantum Hall effect of composite fermions. Phys. Rev. B 66, 155302 (2002).

18. Pinczuk, A. Resonant inelastic light scattering from quantum Hall systems. in Perspectives in Quantum Hall Effects (eds S., Das Sarma \& A., Pinczuk) (Wiley-Interscience, 1997).

19. Park, K. \& Jain, J. K. Two-roton bound state in the fractional quantum Hall effect. Phys. Rev. Lett. 84, 5576-5579 (2000).

20. Ghosh, T. K. \& Baskaran, G. Modeling two-roton bound state formation in the fractional quantum Hall system. Phys. Rev. Lett. 87, 186803 (2001).

21. Kukushkin, I. V., Smet, J. H., Schuh, D., Wegscheider, W. \& von Klitzing, K. Dispersion of the composite-fermion cyclotron-resonance mode. Phys. Rev. Lett. 98, 066403 (2007).

\section{Acknowledgements}

We acknowledge the Computer Centre of IACS for providing its computing facility. D.M. is supported by CSIR, Government of India.

\section{Additional information}

Supplementary information accompanies this paper on www.nature.com/naturephysics. Reprints and permissions information is available online at http://npg.nature.com/ reprintsandpermissions. Correspondence and requests for materials should be addressed to J.K.J. 